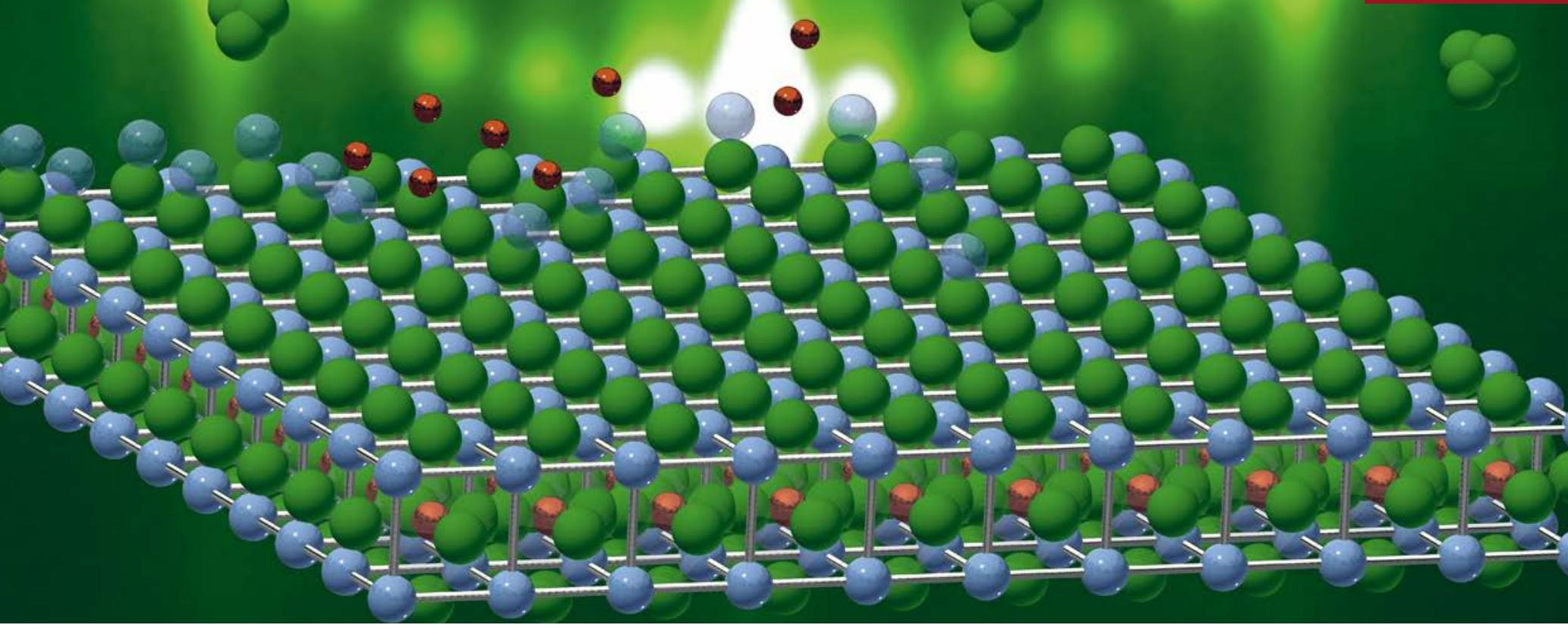

\title{
PRECISE CONTROL OF ATOMS WITH MBE: FROM SEMICONDUCTORS TO COMPLEX OXIDES
}

I Y. Eren Suyolcu ${ }^{1 \star}$, and Gennady Logvenov ${ }^{2 \star}$ - DOI: https://doi.org/10.1051/epn/2020403

- ${ }^{1}$ Department of Materials Science and Engineering, Cornell University, Ithaca, New York 14853, USA

ـ ${ }^{2}$ Max Planck Institute for Solid State Research, Heisenbergstrasse. 1, 70569 Stuttgart, Germany

veren.suyolcu@cornell.edu - g.logvenov@fkf.mpg.de

Molecular Beam Epitaxy (MBE) is a high-vacuum technique with atomic-layer control and precision. It is based on the chemical reaction of the atoms, molecules, or atomic clusters vaporized from the specific evaporation sources on the substrates. The molecular beam defines a unidirectional ballistic flow of atoms and/or molecules without any collisions amongst. In the late 1960s, MBE was initially developed for the growth of $\mathrm{GaAs}$ and $(\mathrm{Al}, \mathrm{Ga})$ As systems $[1,2]$ due to the unprecedented capabilities and then was applied to study other material systems. MBE growth is conventionally performed in vacuum and ultra-high vacuum (UHV) $\left(10^{-8}-10^{-12} \mathrm{mbar}\right)$ conditions.

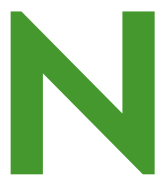

evertheless, additional gases can be assisted in the growth chamber and this MBE is known as reactive $\mathrm{MBE}$ and is mainly used for nitrides or oxides $[3,4]$. Reactive oxide MBE benefits the advantages of both UHV MBE and highly oxidation conditions, while it requires precise gas load control and suitable pumping. Due to the essential requirement of preserving the ballistic path of evaporated atoms, the upper pressure is limited by $\sim 10^{-4} \mathrm{mbar}$ This background pressure determines the choice of the oxidizing gas, for instance, pure ozone injection, which is exceptionally reactive but provides the variety. Similar variety and atomic-layer (AL) precise synthesis can be obtained also with atomic layer deposition (ALD) based on the sequential absorption and desorption of the precursors from the surface[5]. 
FIG 1:

(a) Sketch of an

ozone-assisted MBE

growth chamber.

The abbreviations stand for TP-turbo-

molecular pump,

S-oxide crystal

substrate, RGA-

residual gas analyzer,

Pc1-computer

for controlling the

RHEED system,

Pc2- computer that controls growth itself.

(b) Photo of dual chamber MBE (DCA Instruments $\mathrm{Oy}$ ) in Max Planck Institute

for Solid State

Research [8]

(1) load lock,

(2) storage chamber

(3) central

distribution

chamber, (4) two

growth chambers,

and (5) the ozone

delivery system.

$\rightarrow$ FIG 2:

(a) Sketch of

crystal structures

of Ruddlesden-

Popper (RP)

$A_{n+1} B_{n} \mathbf{O}_{3 n+1}$ phases

with different $n$

( $n=\infty$ corresponds

to cubic perovskite

crystal structure).

(b) Sequence of

the operation of

La and Cu shutters

during single AL-

by-AL growth of

$\mathrm{La}_{2} \mathrm{CuO}_{4}$ ( $\mathrm{RP}$ with

$n=1)$ epitaxial film.

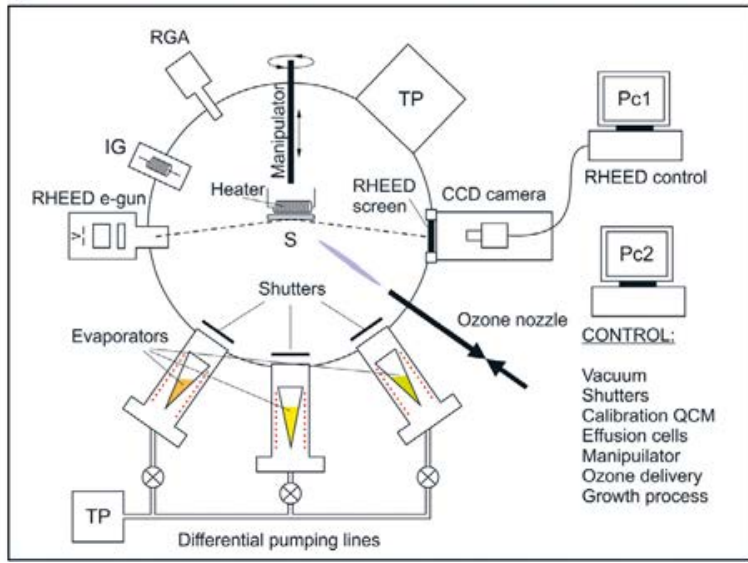

MBE delivers (i) great versatility and flexibility in the target compound selection: An MBE system can be equipped with evaporation sources that could be reloaded with different elements offering variety in synthesizing new compounds; (ii) reliable growth control: MBE systems are equipped with reflection high energy diffraction (RHEED) tools using surface-diffracted electrons to monitor the surface quality in real-time. Together with additional in-situ tools such as low energy electron diffraction (LEED) or angular resolved photoelectron spectroscopy (ARPES) RHEED allows the precise deposition control with low deposition rates (typically one monolayer/min); (iii) atomic layer by atomic layer (AL-by- $\mathrm{AL}$ ) deposition. The selective $\mathrm{AL}$ deposition (with one AL at a time) and the precise control of the concentration of the impurities paves the way for designing functional heterostructures to engineer novel metastable compounds; (iv) the lowest energy of impinging atoms: Different than other physical deposition methods MBE has the lowest energy of impinging atoms $(<0.1 \mathrm{eV})$ providing the lowest undesirable cation intermixing at the interfaces. These superiorities allow versatile heterostructural design with a controlled

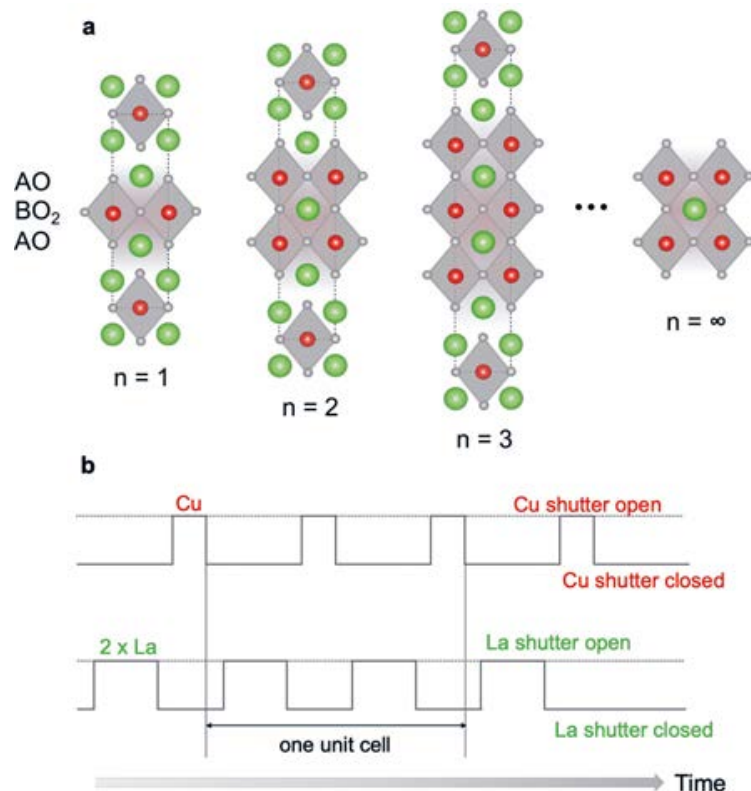

b

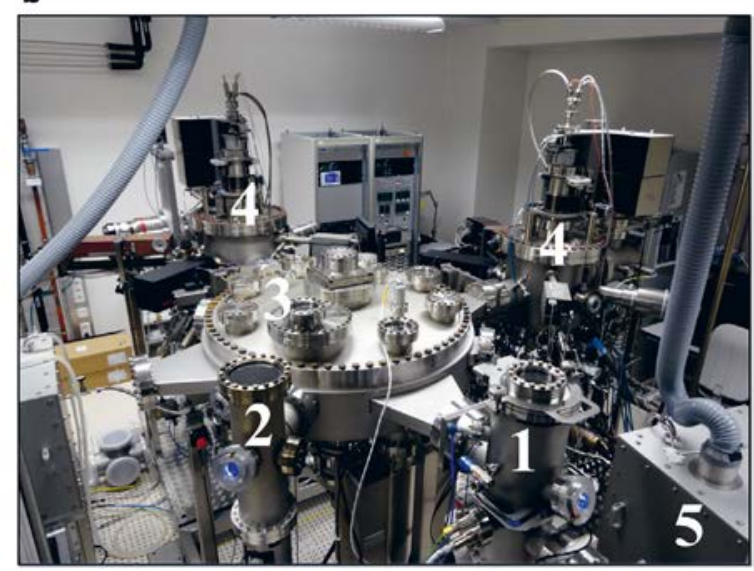

thickness down to a single sheet of atoms resulting in abrupt heterointerfaces[6]. These heterostructures are the building blocks of different kinds of diodes, transistors, including solar cells as well as microprocessors and memory devices. While scaling down the electronics has been the roadmap for semiconductor technology during last more than 50 years[2], the progress in the MBE was stimulated after the discovery of high-temperature superconductivity (HTSC) in 1986 in La-Ba$\mathrm{Cu}-\mathrm{O}$ compound[7], which spiked up the interest to complex oxides family.

Complex oxide materials have ionic crystal structures containing a transition metal (TM) ion centering the unit cell. The layered crystal structure of TM oxide compounds are generally represented by RuddlesdenPopper (RP) phases with $\mathrm{A}_{n+1} \mathrm{~B}_{\mathrm{n}} \mathrm{O}_{3 \mathrm{n}+1}$ formula, where $\mathrm{A}$ represents alkali, alkaline earth, or rare earth metal, $B$ is the TM, and $\mathrm{n}$ - integer number. These RP phases consist of two-dimensional perovskite-like slabs (Figure 2a) that can be precisely designed by MBE[6]. The AL-by-AL method is best suited for the fabrication layered oxides, where the precise counting of the constituent atoms is realized via shuttering. Shuttering times are individually determined according to the flux emanating from each elemental source. For instance, in the case of synthesis of $\mathrm{La}_{2} \mathrm{CuO}_{4}$, which has $\mathrm{RP}$ crystal structure with $\mathrm{n}=1$ (represented in Figure $2 \mathrm{a}: \mathrm{A}=\mathrm{La}$ and $\mathrm{B}=\mathrm{Cu}$ ) the sequence of operation of $\mathrm{La}$ and $\mathrm{Cu}$ shutters are represented in Figure $2 \mathrm{~b}$. The time between the shutter open and close is computer controlled and the whole process is monitored by in-situ RHEED with real-time feedback.

The adaptable perovskite structure of TM oxides offers constructing them in different forms, such as ultrathin films or heterostructures[9], but also leads to diverse physical properties ranging from HTSC[10-12] to thermoelectricity[13]. In other words, engineering of epitaxial oxides grants unlimited combinations of multilayers delivering a fundamental playground for device fabrication and possible applications. A multilayer, e.g. a superlattice, composed of the same material with same crystal structure (even with modulation doping) 
is an example of homo-epitaxial growth, while a multilayer built by stacking of materials with different chemical formula and crystal structure, this is an example of hetero-epitaxial growth.

The strong electron correlations at the designed heterointerfaces emerge novel interfacial properties when different materials are attached adjacently. Thus, interface engineering is an intriguing yet challenging task and the atomically precise design of complex oxide heterostructures requires atomic-resolution identification of individual layers and interfaces (Figure 3). The prominent role is played by aberration-corrected scanning transition electron microscopy (STEM) with high-resolution imaging and spectroscopy capabilities[14,15]. STEM high-angle annular dark field (HAADF) images of three different $\mathrm{La}_{2} \mathrm{CuO}_{4}$-based heterostructures are presented in Figure $3 a-c[6]$ displaying the ideally arranged crystal structure without any extended defects.

In conclusion, besides of high-quality semiconductor fabrication MBE can also be effectively used for synthesis of difficult compounds, e.g. complex oxides with a crystal quality analogous to the semiconductor multilayers while the interfaces even more abrupt. Complex oxides have been widely studied recently and even though it requires time to put the new substances to practical use, a substantial progress has been already established.

\section{About the Authors}

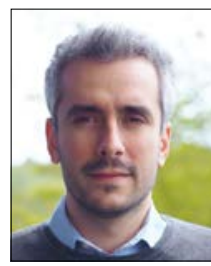

Y. Eren Suyolcu is a Postdoctoral Associate at Cornell University, New York, USA.After completing his $\mathrm{PhD}$ research at the Max Planck Institute for Solid State Research,Stuttgart, Germany,Dr.Suyolcu joined Cornell University and continues his research on oxide-MBE growth and aberration-corrected STEM of oxide heterostructures and interfaces.

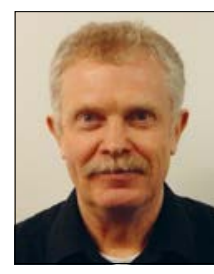

Gennady Logvenov is the Head of Thin Film Scientific Facility at Max Planck Institute for Solid State Research, Stuttgart, Germany. After several experiences in world-class international institutions, Dr. Logvenov has been leading his group since 2011 and fabricating highquality oxide heterostructures.

\section{References}

[1] A. Y. Cho, Journal of Applied Physics 41, 2780 (1970).

[2] M. A. Herman, H. Sitter, Molecular Beam Epitaxy: Fundamentals and Current Status, Springer-Verlag, Berlin Heidelberg (1989).

[3] S. Yoshida, S. Misawa, Y. Fujii, S. Takada, H. Hayakawa, S. Gonda, A. Itoh, Journal of Vacuum Science and Technology 16, 990 (1979).

[4] D. G. Schlom, L.-Q. Chen, X. Pan, A. Schmehl, M. A. Zurbuchen, Journal of the American Ceramic Society 91, 2429 (2008).

[5] S. M. George, Chem. Rev. 2010, 110, 111.

[6] Y. E. Suyolcu, G. Christiani, P. A. van Aken, G. Logvenov, J Supercond Nov Magn 33, 107 (2020).

[7] J. G. Bednorz, K. A. Müller, Z. Physik B - Condensed Matter 64 189 (1986).

[8] F. Baiutti, F. Wrobel, G. Christiani, G. Logvenov, in Metal Oxide-Based Thin Film Structures (Eds.: N. Pryds, V. Esposito), Elsevier, 53 (2018).

[9] R. Ramesh, D. G. Schlom, Nature Reviews Materials, 1 (2019).

[10] A. Gozar, G. Logvenov, L. F. Kourkoutis, A. T. Bollinger, L. A. Giannuzzi, D. A. Muller, I. Bozovic, Nature 2008, 455, 782.

[11] Y. E. Suyolcu, Y. Wang, F. Baiutti, A. Al-Temimy, G. Gregori, G. Cristiani, W. Sigle, J. Maier, P. A. van Aken, G. Logvenov, Scientific Reports 7, 453 (2017).

[12] F. Baiutti, G. Gregori, Y. E. Suyolcu, Y. Wang, G. Cristiani, W. Sigle, P. A. van Aken, G. Logvenov, J. Maier, Nanoscale 10, 8712 (2018).

[13] P. Kaya, G. Gregori, F. Baiutti, P. Yordanov, Y. E. Suyolcu, G. Cristiani, F. Wrobel, E. Benckiser, B. Keimer, P. A. van Aken H.-U. Habermeier, G. Logvenov, J. Maier, ACS Appl. Mater. Interfaces 10, 22786 (2018).

[14] Y. E. Suyolcu, Y. Wang, W. Sigle, F. Baiutti, G. Cristiani, G. Logvenov, J. Maier, P. A. van Aken, Adv. Mater. Interfaces 4 , 1700737 (2017).

[15] Y. E. Suyolcu, Y. Wang, F. Baiutti, W. Sigle, G. Cristiani, G. Gregori, G. Logvenov, J. Maier, P. A. van Aken, JEOL News 53, 8 (2018).

[16] G. Kim, Y. Khaydukov, M. Bluschke, Y. E. Suyolcu, G. Christiani, K. Son, C. Dietl, T. Keller, E. Weschke, P. A. van Aken, G. Logvenov, B. Keimer, Phys. Rev. Materials 3, 084420 (2019). a

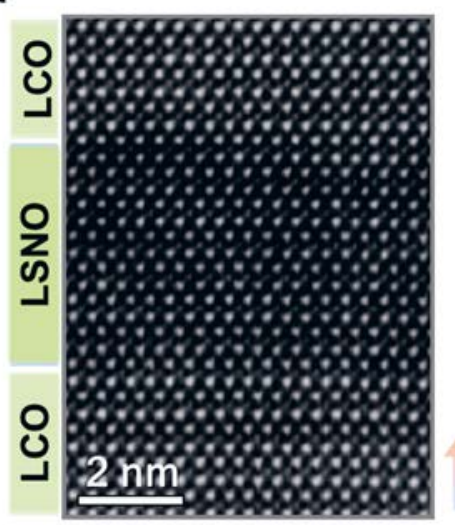

b

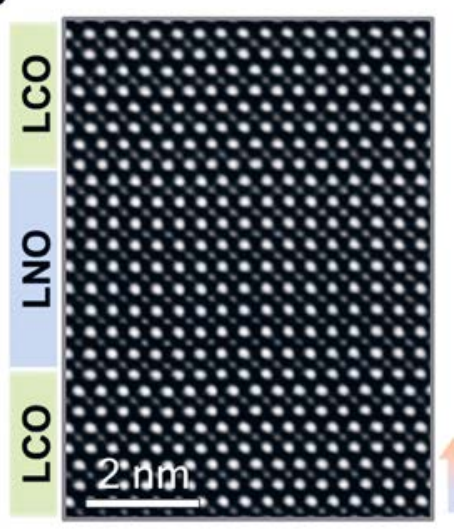

c

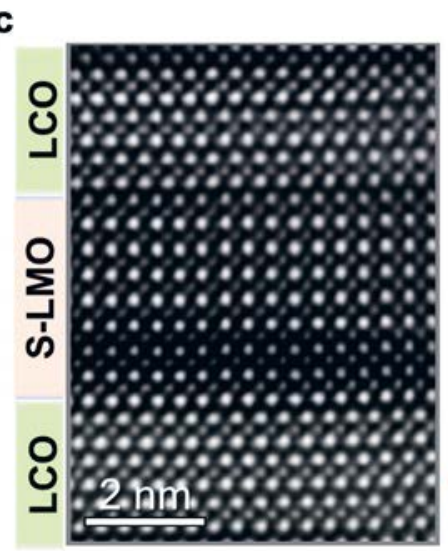

4 FIG 3: Example STEM-HAADF

images showing the structural coherency between the layers of (a) LCO-LSNO-LCO [12], (b) LCO-LNO-LGO [13], (c) LCO-SMO/LMOLCO [16] grown on (001) LSAO crystal substrates [6]. The abbreviations stand for $\mathrm{LCO}-\mathrm{La}_{2} \mathrm{CuO}_{4}$, LSNO - $\mathrm{La}_{2-\mathrm{S}} \mathrm{Sr}_{\mathrm{x}} \mathrm{NiO}_{4}$, $\mathrm{SMO}-\mathrm{SrMnO}_{3}$ and $\mathrm{LMO}-\mathrm{LaMnO}_{3}$ [6]. 